\title{
Three-Mask Polycrystalline Silicon TFT With Metallic Gate and Junctions
}

\author{
Dongli Zhang and Man Wong, Senior Member, IEEE
}

\begin{abstract}
Polycrystalline silicon thin-film transistors (TFTs) with metallic gates and junctions realized using a three-mask metal-replaced junction (MERJ) technology have been fabricated and characterized. Compared to those of a conventional TFT, the process of making a MERJ TFT is simplified, and the resistance of the junctions and gate is reduced. The low resistance of the metallic junctions allows a greater recovery of the intrinsic characteristics of a MERJ TFT, and the reduced signal delay on a low-resistance metallic gate line makes the TFT particularly suitable for realizing large-area active-matrix flat-panel displays.
\end{abstract}

Index Terms-Aluminum (Al), flat-panel display, metal gate, metal-replaced junction (MERJ), polycrystalline silicon (poly-Si), thin-film transistor (TFT).

\section{INTRODUCTION}

$\mathbf{T}$ HOUGH most active-matrix flat-panel displays are fabricated using amorphous-silicon (a-Si) thin-film transistors (TFTs) because of the simplicity of the technology [1], the relatively low field-effect mobility $\left(\mu_{\mathrm{FE}}\right)$ of a-Si TFTs limits their application to only the realization of the pixel switches [2]. Further integration of drivers and other circuits requires TFTs with higher $\mu_{\mathrm{FE}}$, such as those based on polycrystalline silicon (poly-Si) [3], [4].

In terms of mask count and process complexity, the construction of circuits based on poly-Si TFTs is comparable to that based on bulk Si transistors and requires a minimum of four masking steps. The mask count is at least $50 \%$ higher for circuits containing complementary n- and p-type TFTs. For poly-Si TFTs fabricated on glass, the problem of high junction resistance is aggravated by the reduced dopant activation at the typically low process temperature [5]. High parasitic resistance degrades the apparent performance of a TFT [6].

The replacement of poly-Si with aluminum (Al) has been previously applied to the formation of high-aspectratio contact plugs [7] and the gate electrode of a bulk metal-oxide-semiconductor transistor [8]. It is more recently applied to the formation of $\mathrm{p}$-channel poly-Si TFTs with metal-replaced junctions (MERJs) [9]. The construction of active-matrix displays based only on p-channel TFTs has been proposed [10] because of higher device reliability [11] and reduced process complexity. A simple three-mask MERJ process

Manuscript received March 6, 2006; revised April 12, 2006. This work was supported by a grant from the Research Grants Council of the Hong Kong Special Administrative Region. The review of this letter was arranged by Editor Y. Taur.

The authors are with the Department of Electrical and Electronic Engineering, Hong Kong University of Science and Technology, Kowloon, Hong Kong (e-mail: eemwong@ee.ust.hk).

Digital Object Identifier 10.1109/LED.2006.876309

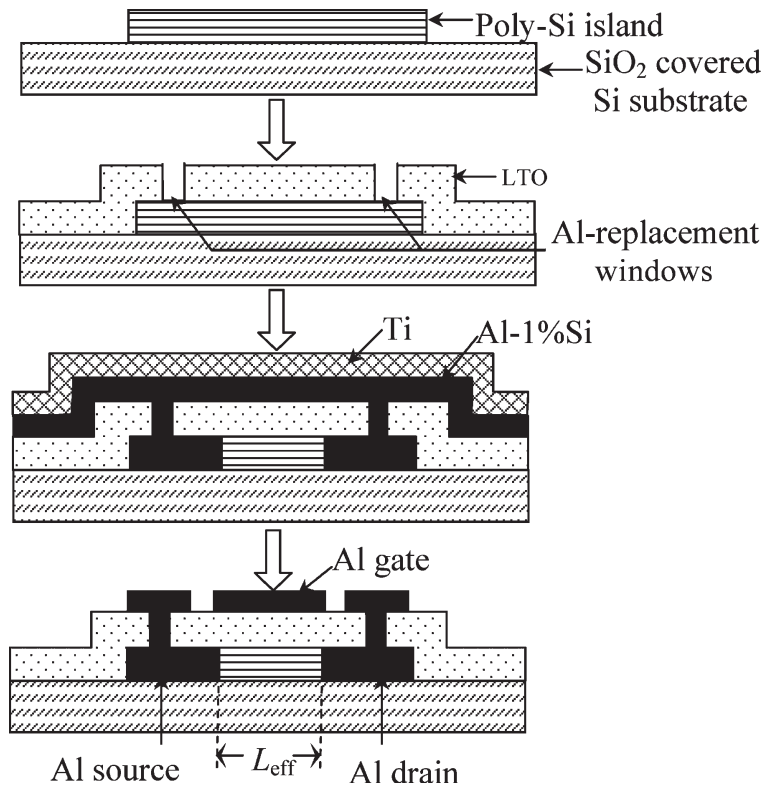

Fig. 1. Schematic diagrams showing the evolution of the device cross section during the fabrication of a three-mask MERJ TFT. $L_{\text {eff }}$ is the effective channel length.

is presently proposed to realize a poly-Si TFT with metallic gate, source, and drain. The low-resistance metallic source and drain junctions allow a significant recovery of the intrinsic characteristics of a MERJ TFT. The signal delay on the long "scan" lines that are used to drive the rows of the gates of the pixel switch TFTs in an active-matrix panel is roughly proportional to the product of the total line resistance and line capacitance. The reduced delay on a low-resistance metallic gate line makes the MERJ TFTs particularly suitable for realizing large-area active-matrix panels.

\section{DEVICE FABRICATION}

As starting substrates, 100-mm-diameter (100)-oriented Si wafers covered with 500-nm thermally grown Si dioxide were used. A 35-nm-thick a-Si layer was deposited by low-pressure chemical vapor deposition (LPCVD) at $550{ }^{\circ} \mathrm{C}$ and patterned to form the active islands before the conventional nickelbased metal-induced crystallization (MIC) [12] at $550{ }^{\circ} \mathrm{C}$ was performed. After the deposition of a 70-nm-thick LPCVD low-temperature oxide (LTO) at $450{ }^{\circ} \mathrm{C}$ as the gate insulator layer, the $\mathrm{Al}$ replacement windows (Fig. 1) were opened. This was followed by a sequential deposition of a double layer of 2- $\mu$ m-thick Al-1\%Si alloy and 300-nm-thick titanium (Ti) by sputtering. 


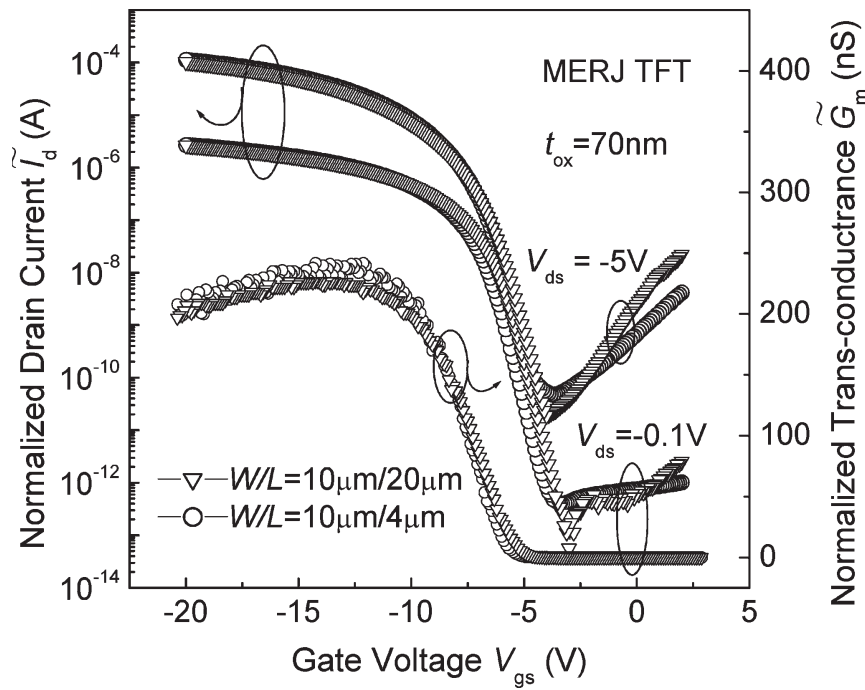

Fig. 2. Normalized drain-current $\widetilde{I}_{\mathrm{d}}$ and normalized transconductance $\widetilde{G}_{m}$ versus $V_{\mathrm{gs}}$ characteristics of MERJ TFTs at $V_{\mathrm{ds}}=-0.1$ and $-5 \mathrm{~V}$. $t_{\mathrm{ox}}$ is the gate oxide thickness, $W$ is the drawn channel width, and $L$ is the drawn channel length.

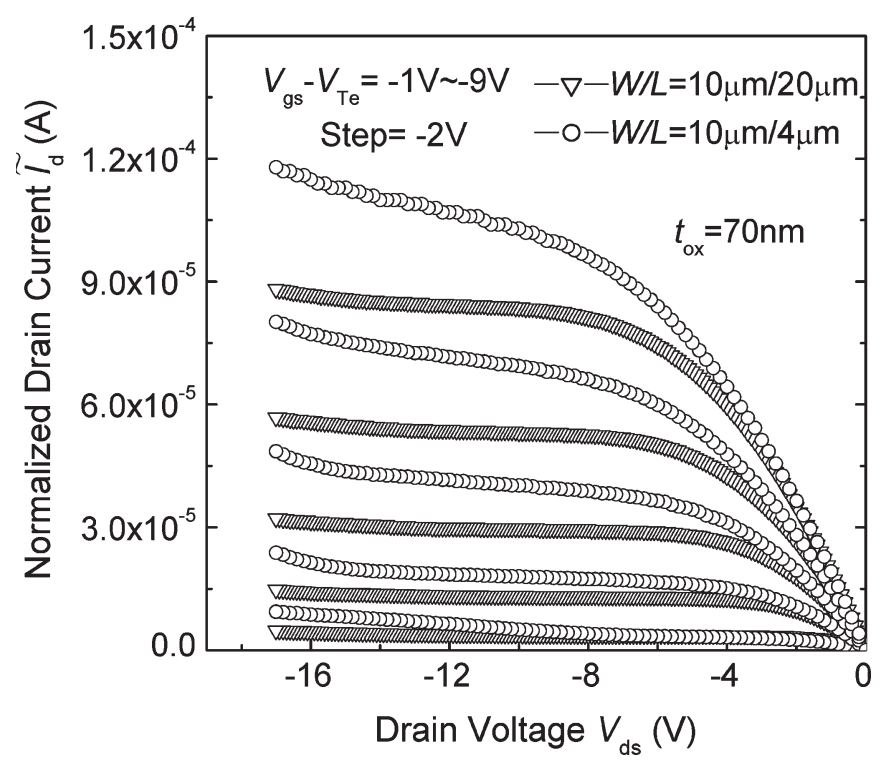

Fig. 3. Normalized drain-current $\widetilde{I}_{\mathrm{d}}$ versus $V_{\mathrm{ds}}$ characteristics of MERJ TFTs at different values of the gate drive $V_{\mathrm{gs}}-V_{\mathrm{Te}}$.

After heat treatment at $400{ }^{\circ} \mathrm{C}$ for $2.5 \mathrm{~h}$ in atmospheric pressure nitrogen, the nominally undoped poly-Si in the vicinity of the replacement windows was replaced by $\mathrm{Al}$ at a rate of $\sim 2.5 \mu \mathrm{m} / \mathrm{h}$. The replacement $\mathrm{Al}$ eventually formed the source and drain junctions of a MERJ TFT. Any remaining Ti was removed in concentrated hydrofluoric acid. Finally, the Al layer was patterned to form the interconnections and the gate electrodes. The resulting TFTs were characterized using an HP 4156 semiconductor parameter analyzer.

The realization of a conventional poly-Si TFT requires at least four masks and a heat treatment step to activate the implanted junctions. With the use of only three masks and the elimination of the dopant-activation step, both the complexity and the temperature of making a MERJ TFT are greatly reduced. Similar to the case of the conventional a-Si TFT

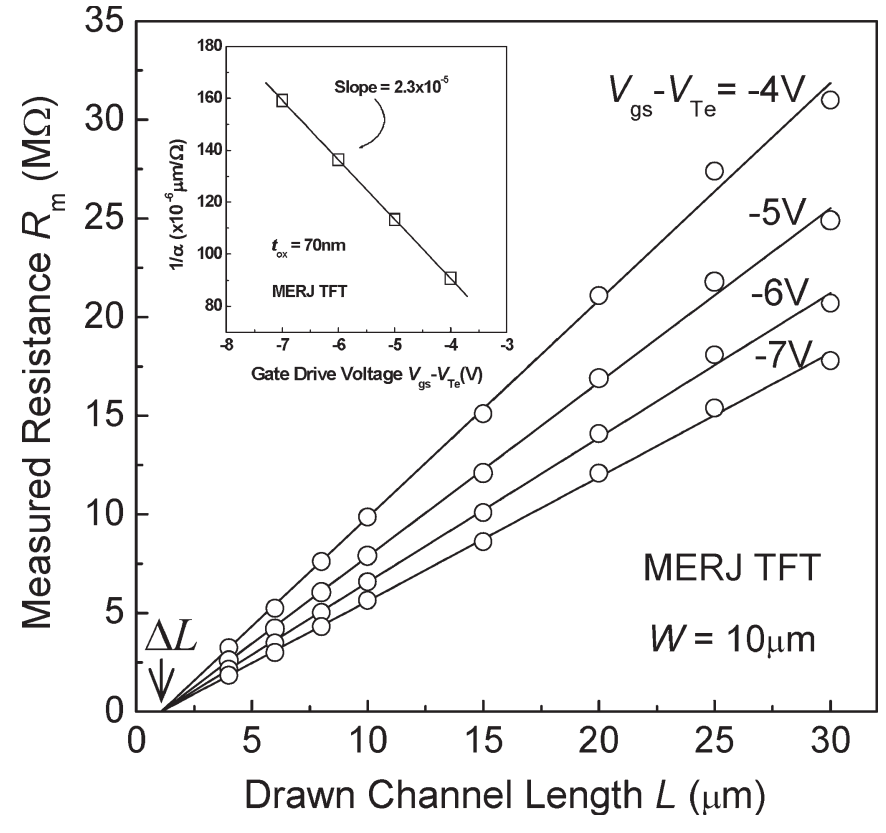

Fig. 4. Measured $R_{m} \equiv V_{\mathrm{ds}} / I_{\mathrm{d}}$ versus $L$ of a series of MERJ TFTs at different values of the gate drive $V_{\mathrm{gs}}-V_{\mathrm{Te}}$. The slopes of the lines and their dependence on $V_{\mathrm{gs}}-V_{\mathrm{Te}}$ are shown in the inset. $W$ is $10 \mu \mathrm{m}$ for all of the TFTs.

technology, the source and drain junctions are not self-aligned to the gate electrode. The amount of the misalignment between the gate and the junctions is controlled by the overlay accuracy.

\section{RESUlts AND Discussion}

The sheet resistance of the resulting $\mathrm{Al}$ gate is measured to be $\sim 0.02 \Omega$, with a corresponding resistivity of $\sim 4 \mu \Omega \cdot \mathrm{cm}$ that is close to the intrinsic resistivity of Al. The sheet resistance of the $\mathrm{Al}$ source and drain of a MERJ TFT is $\sim 2 \Omega$, which corresponds to a resistivity of $\sim 7 \mu \Omega \cdot \mathrm{cm}$ that is approximately double the intrinsic resistivity of Al. Similar increase in resistivity, which is attributed to the reduced purity of the replacement $\mathrm{Al}$, has been reported [13]. The total parasitic source and drain resistance of a MERJ TFT is $\sim 1.6 \Omega$.

Shown in Figs. 2 and 3 are the respective normalized drain-current $\widetilde{I}_{\mathrm{d}} \equiv I_{\mathrm{d}}\left(L_{\mathrm{eff}} / W\right)$ versus $V_{\mathrm{gs}}$ and $\widetilde{I}_{\mathrm{d}}$ versus $V_{\mathrm{ds}}$ characteristics of MERJ TFTs with drawn channel lengths $L$ of 4 and $20 \mu \mathrm{m} . I_{\mathrm{d}}$ is the drain-current, $V_{\mathrm{gs}}$ is the terminal gate-tosource voltage, $V_{\mathrm{ds}}$ is the terminal drain-to-source voltage, $W$ is the channel width, and $L_{\text {eff }}$ is the effective channel length. Because of the diffusion of $\mathrm{Al}$ [14], which is an acceptor in $\mathrm{Si}$ [15], into the channel regions adjacent to the metallic $\mathrm{Al}$ junctions, the MERJ TFTs exhibit typical p-channel device characteristics even without any deliberate junction doping.

Obtained from the transfer characteristics (Fig. 2) of the $L=20 \mu \mathrm{m}$ TFT in Fig. 2 are a linearly extrapolated threshold voltage $V_{\mathrm{Te}}$ of $\sim-8 \mathrm{~V}$, a subthreshold swing of $\sim 0.6 \mathrm{~V} / \mathrm{dec}$, a minimum leakage current $I_{\mathrm{off}}$ of $\sim 10 \mathrm{pA}$ at $V_{\mathrm{ds}}=-5 \mathrm{~V}$, and an on-current $\left(I_{\mathrm{d}}\right.$ at $\left.V_{\mathrm{gs}}=-20 \mathrm{~V}\right)$ to $I_{\text {off }}$ ratio of $\sim 5.5 \times 10^{6}$ at $V_{\mathrm{ds}}=-5 \mathrm{~V}$. From the maximum apparent normalized transconductance $\left.\widetilde{G}_{m} \equiv\left(\partial \widetilde{I}_{\mathrm{d}} / \partial V_{\mathrm{gs}}\right)\right|_{V_{\mathrm{ds}}=-0.1 \mathrm{~V}}$ versus $V_{\mathrm{gs}}$ characteristics in Fig. 2 and without correcting for the parasitic 
junction resistance, an apparent $\mu_{\mathrm{FE}}$ of $\sim 45.5 \mathrm{~cm}^{2} / \mathrm{V} \cdot \mathrm{s}$ is extracted.

Fig. 4 is generated using the data extracted from the transfer characteristics of a series of MERJ TFTs at $V_{\mathrm{ds}}=-0.05 \mathrm{~V}$. A process-induced channel length reduction $\Delta L \equiv L-L_{\text {eff }}$ of $\sim 1.2 \mu \mathrm{m}$ and an "intrinsic" $\mu_{\mathrm{FE}}$ of $\sim 46.3 \mathrm{~cm}^{2} / \mathrm{V} \cdot \mathrm{s}$ can be extracted using the procedure outlined in [5]. The latter is only slightly higher than the apparent $\mu_{\mathrm{FE}}$ of $\sim 45.5 \mathrm{~cm}^{2} / \mathrm{V} \cdot \mathrm{s}$, thus verifying the insignificant impact of the low parasitic junction resistance of $\sim 1.6 \Omega$.

\section{CONCLUSION}

Poly-Si TFTs with metal gate and junctions realized using a simple three-mask MERJ technology have been fabricated and characterized. The resistance of the gate and the junctions is greatly reduced. The low parasitic junction resistance makes it possible to unmask the intrinsic characteristics of a MERJ TFT.

\section{REFERENCES}

[1] T. Tsukada, "Active-matrix liquid-crystal displays," in Technology and Applications of Hydrogenated Amorphous Silicon, R. A. Street, Ed. New York: Springer-Verlag, 2000, pp. 7-93.

[2] H. Yamamoto, H. Matsumaru, K. Shirahashi, M. Nakatani, A. Sasano, N. Konishi, K. Tsutsui, and T. Tsukada, "A new a-Si TFT with $\mathrm{Al}_{2} \mathrm{O}_{3} / \mathrm{SiN}$ double-layered gate insulator for 10.4-inch diagonal multicolor display," in IEDM Tech. Dig., 1990, pp. 851-854.

[3] W. Czubatyj, D. Beglau, R. Himmler, G. Wicker, D. Jablonski, and S. Guha, "Low-temperature polycrystalline-silicon TFT on 7059 glass," IEEE Electron Device Lett., vol. 10, no. 8, pp. 349-351, Aug. 1989.

[4] Z. Meng, M. Wang, and M. Wong, "High performance low temperature metal-induced unilaterally crystallized polycrystalline silicon thin film transistors for system-on-panel applications," IEEE Trans. Electron Devices, vol. 47, no. 2, pp. 404-409, Feb. 2000.

[5] D. L. Zhang and M. Wong, "Effects of trace nickel on the growth kinetics and the electrical characteristics of metal-induced laterally crystallized polycrystalline silicon and devices," J. Soc. Inf. Disp, vol. 13, no. 10, pp. 815-822, Oct. 2005.

[6] S.-C. Chang, C.-C. Wu, I.-M. Lu, and Y.-E Chen, "Effect of parasitic resistance on mobility in laser crystallized LT poly-Si TFTs," in Proc. ASID, Hsinchu, Taiwan, 1999, pp. 293-296.

[7] H. Horie, M. Imai, A. Itoh, and Y. Arimoto, "Novel high aspect ratio aluminum plug for logic/DRAM LSIs using polysilicon-aluminum substitute (PAS)," in IEDM Tech. Dig., 1996, pp. 946-948.

[8] Y. Mishima, H. Shido, T. Kurahashi, T. Nagata, J. Naganuma, H. Kudo, and S. Nakamura, "Characteristics of aluminum substitution technology for self-aligned full metal gate nMOSFETs," IEEE Trans. Electron Devices, vol. 52, no. 5, pp. 962-966, May 2005.

[9] D. L. Zhang and M. Wong, "Metal-replaced junction (MERJ) for reducing the junction parasitic resistance of a thin-film transistor," IEEE Electron Device Lett., vol. 27, no. 4, pp. 269-271, Apr. 2006.

[10] S.-H Jung, W.-J Nam, C.-W. Han, and M.-K. Han, "A new low power PMOS poly-Si inverter and driving circuits for active-matrix displays," in Proc. SID Dig., 2003, pp. 1396-1399.

[11] Y. Uraoka, T. Hatayama, T. Fuyuki, T. Kawamura, and Y. Tsuchihashi, "Reliability of low temperature poly-silicon TFTs under inverter operation," IEEE Trans. Electron Devices, vol. 48, no. 10, pp. 2370-2374, Oct. 2001.

[12] S. Y. Yoon, K. H. Kim, C. O. Kim, J. Y. Oh, and J. Jang, "Low temperature metal induced crystallization of amorphous silicon using nickel solution," J. Appl. Phys., vol. 82, no. 22, pp. 5865-5867, 1997.

[13] S. Nakamura, R. Suzuki, M. Fukuda, M. Kobayashi, and A. Hatada, "Aluminum word line and bit line fabrication technology for COB DRAM using a polysilicon-aluminum substitute," in VLSI Symp. Tech. Dig., 1999, pp. 35-36.

[14] K. Nakamura and M. Kamoshida, "Low-temperature diffusion of Al into polycrystalline Si," J. Appl. Phys., vol. 48, no. 12, pp. 5349-5351, Dec. 1977.

[15] B.-Y. Tsaur, G. W. Turner, and J. C. C. Fan, "Efficient Si solar cells by low-temperature solid phase epitaxy," Appl. Phys. Lett., vol. 39, no. 9, pp. 749-751, Nov. 1981. 